TRANSACTIONS OF THE

AMERICAN MATHEMATICAL SOCIETY

Volume 349, Number 10, October 1997, Pages 4231-4249

S 0002-9947(97)01947-8

\title{
EXISTENCE OF POSITIVE SOLUTIONS FOR SOME PROBLEMS WITH NONLINEAR DIFFUSION
}

\author{
A. CAÑADA, P. DRÁBEK, AND J. L. GÁMEZ
}

\begin{abstract}
In this paper we study the existence of positive solutions for problems of the type

$$
-\Delta_{p} u(x)=u(x)^{q-1} h(x, u(x)), \quad x \in \Omega
$$$$
u(x)=0, \quad x \in \partial \Omega,
$$

where $\Delta_{p}$ is the $p$-Laplace operator and $p, q>1$. If $p=2$, such problems arise in population dynamics. Making use of different methods (sub- and supersolutions and a variational approach), we treat the cases $p=q, p<q$ and $p>q$, respectively. Also, some systems of equations are considered.
\end{abstract}

\section{INTRODUCTION}

In this paper we consider problems of the type

$$
\begin{array}{cl}
-\Delta_{p} u(x)=u(x)^{q-1} h(x, u(x)), & x \in \Omega, \\
u(x)=0, & x \in \partial \Omega,
\end{array}
$$

where $\Omega$ is a bounded and regular domain in $\mathbb{R}^{N}, p, q$ are real constants, $p, q>1, \Delta_{p}$ is the $p$-Laplace operator $\Delta_{p} u=\operatorname{div}\left(|\nabla u|^{p-2} \nabla u\right)$, and $h$ is a real function defined on $\bar{\Omega} \times \mathbb{R}$, satisfying certain weak regularity conditions (see (H1-H2) in the next section).

Problem (1.1) generalizes those arising in population dynamics, where usually $p=2$, the function $u$ represents the density (at different points of $\Omega$ ) of a biological species, and, depending on the value of $q$, the problem has "linear diffusion" (if $q=2$ ), "slow diffusion" (if $q<2$ ), or "fast diffusion" (if $q>2$ ). Results for the case $q \leq p=2$ (linear or slow diffusion) can be found in [4], [7], [11].

The case of "fast diffusion" $(q>p=2)$ is not widely treated in the literature, even though it is interesting. One reason for this is that the methods used for the other cases do not generally work well for it.

Received by the editors October 11, 1994 and, in revised form, May 6, 1996.

1991 Mathematics Subject Classification. Primary 35J65, 35J55; Secondary 47H17, 58E30, 92D25.

Key words and phrases. Nonlinear elliptic problems, boundary value problems, positive solutions, nonlinear diffusion, sub- and super-solutions, variational methods.

The first and the third author have been supported in part by DGICYT, Ministry of Education and Science (Spain), under grant number PB95-1190 and by EEC contract, Human Capital and Mobility program, ERBCHRXCT940494. The second author was partially supported by the Grant Agency of the Czech Republic under Grant No. 201/94/0008, and he is grateful to University of Granada for pleasant hospitality during preparation of this paper.

(C)1997 American Mathematical Society 
Here we shall consider arbitrary $p>1$. We will denote by $p^{\prime}$ the exponent conjugate to $p$, i.e. $\frac{1}{p}+\frac{1}{p^{\prime}}=1$, and by $p^{*}$ the critical Sobolev exponent, i.e. $p^{*}=\frac{N p}{N-p}$ if $p<N, p^{*}=+\infty$ if $p \geq N$.

The existence results for (1.1) are obtained in Section 3 for the cases $1<q<p$, $q=p$ and $p<q<p^{*}$ (see Theorem 3.1). The main tool is a general method of sub- and super-solutions described in Section 2. Trying to cover also the case with general $q>p$, we develop a variational-type method which allows us to obtain an existence result for (1.1) even for $q \geq p^{*}$ (see Theorem 3.2) under slightly different assumptions on $h=h(x, s)$.

Finally, in Section 4, we consider general systems of the form

$$
\begin{array}{cl}
-\Delta_{p} u(x)=u(x)^{q-1} h(x, u(x), v(x)), & x \in \Omega, \\
-\Delta_{p} v(x)=v(x)^{q-1} k(x, u(x), v(x)), & x \in \Omega, \\
u(x)=v(x)=0, & x \in \partial \Omega,
\end{array}
$$

and we develop a method of sub- and super-solutions for such systems, similar to those given in [5], [7], [8]. As an application of that method, we give different conditions to obtain a coexistence result for a particular system in the case $p<q<$ $p^{*}$, and in the general case $p<q$.

2. The Method of Sub- And Super-SOlutions. The PRincipal EIgenvalue OF THE $p$-LAPLACIAN WITH A WEIGHT FUNCTION

Consider the problem

$$
\begin{array}{cl}
-\Delta_{p} u(x)=\widetilde{h}(x, u(x)), & x \in \Omega, \\
u(x)=0, & x \in \partial \Omega,
\end{array}
$$

where $\Omega$ is a bounded and regular domain in $\mathbb{R}^{N}$ and $\widetilde{h}: \bar{\Omega} \times \mathbb{R} \rightarrow \mathbb{R}$ satisfies the following hypotheses:

(H1)

$\widetilde{h}(x, s)$ is a Carathéodory function (i.e. $\widetilde{h}(x,$.$) is continuous for$ a.e. $x \in \Omega$ and $\widetilde{h}(\cdot, s)$ is measurable for all $s \in \mathbb{R})$ and $\widetilde{h}(\cdot, s)$ is bounded if $s$ belongs to bounded sets.

$$
\begin{aligned}
& \exists g: \mathbb{R} \rightarrow \mathbb{R}, \text { continuous and increasing, such that } g(0)=0 \text {, and } \\
& \text { the map } s \mapsto \widetilde{h}(x, s)+g(s) \text { is nondecreasing, for a.e. } x \in \Omega \text {. }
\end{aligned}
$$

Definition 2.1. A function $\underline{u} \in W^{1, p}(\Omega) \cap L^{\infty}(\Omega)$ is said to be a sub-solution of (2.1) if

$$
\begin{cases}\int_{\Omega}|\nabla \underline{u}|^{p-2} \nabla \underline{u} \cdot \nabla \phi \leq \int_{\Omega} \widetilde{h}(x, \underline{u}) \phi, & \forall \phi \in W_{0}^{1, p}(\Omega), \phi \geq 0, \\ \underline{u} \leq 0 & \text { on } \partial \Omega .\end{cases}
$$

(A function $v \in W^{1, p}(\Omega)$ is said to be less than or equal to $w \in W^{1, p}(\Omega)$ on $\partial \Omega$ when $\max \{0, v-w\} \in W_{0}^{1, p}(\Omega)$.) 
In the same way, a function $\bar{u} \in W^{1, p}(\Omega) \cap L^{\infty}(\Omega)$ is said to be a super-solution of $(2.1)$ if

$$
\begin{cases}\int_{\Omega}|\nabla \bar{u}|^{p-2} \nabla \bar{u} \cdot \nabla \phi \geq \int_{\Omega} \widetilde{h}(x, \bar{u}) \phi, & \forall \phi \in W_{0}^{1, p}(\Omega), \phi \geq 0, \\ \bar{u} \geq 0 & \text { on } \partial \Omega .\end{cases}
$$

Lemma 2.2 (Maximum Principle). Consider $g$ from (H2) and functions $u_{1}, u_{2} \in$ $W^{1, p}(\Omega) \cap L^{\infty}(\Omega)$ such that, $\forall \phi \in W_{0}^{1, p}(\Omega), \phi \geq 0$ :

$$
\left\{\begin{array}{l}
\int_{\Omega}\left|\nabla u_{2}\right|^{p-2} \nabla u_{2} \cdot \nabla \phi+\int_{\Omega} g\left(u_{2}\right) \phi \leq \int_{\Omega}\left|\nabla u_{1}\right|^{p-2} \nabla u_{1} \cdot \nabla \phi+\int_{\Omega} g\left(u_{1}\right) \phi \\
\text { and } u_{2} \leq u_{1} \text { on } \partial \Omega .
\end{array}\right.
$$

Then $u_{2} \leq u_{1}$ a.e. in $\Omega$.

Proof. Let us choose $\phi=\left(u_{2}-u_{1}\right)^{+} \in W_{0}^{1, p}(\Omega)$. Then it follows from (H2) that

$$
\begin{aligned}
0 \geq & \int_{\Omega}\left[\left|\nabla u_{2}\right|^{p-2} \nabla u_{2}-\left|\nabla u_{1}\right|^{p-2} \nabla u_{1}\right] \cdot \nabla\left(u_{2}-u_{1}\right)^{+} \\
& +\int_{\Omega}\left[g\left(u_{2}\right)-g\left(u_{1}\right)\right]\left(u_{2}-u_{1}\right)^{+} \\
= & \int_{\Omega} \frac{\left|\nabla u_{2}\right|^{p-2}+\left|\nabla u_{1}\right|^{p-2}\left|\nabla\left(u_{2}-u_{1}\right)^{+}\right|^{2}}{2}\left[\left|\nabla u_{2}\right|^{2}-\left|\nabla u_{1}\right|^{2}\right] \\
& +\int_{\left\{u_{2} \geq u_{1}\right\}} \frac{\left|\nabla u_{2}\right|^{p-2}-\left|\nabla u_{1}\right|^{p-2}}{2}\left[g\left(u_{2}\right)-g\left(u_{1}\right)\right]\left(u_{2}-u_{1}\right)^{+} .
\end{aligned}
$$

Observe that every summand in this last expression is nonnegative, and hence we obtain that $\left(u_{2}-u_{1}\right)^{+}=0$ a.e. in $\Omega$ or, equivalently, $u_{2} \leq u_{1}$ a.e. in $\Omega$.

Lemma $2.3([6])$. For every function $f \in L^{p^{\prime}}(\Omega)$, the problem

$$
\begin{array}{cl}
-\Delta_{p} u(x)+g(u(x))=f(x), & x \in \Omega, \\
u(x)=0, & x \in \partial \Omega,
\end{array}
$$

admits a unique weak solution $u \in W_{0}^{1, p}(\Omega)$. Moreover, the associated operator $T: L^{p^{\prime}}(\Omega) \rightarrow W_{0}^{1, p}(\Omega), f \mapsto u$ is continuous and nondecreasing.

The proof of this result can be found in [6]. The fact that $T$ is nondecreasing follows from Lemma 2.2 .

Theorem 2.4. Consider problem (2.1), under hypotheses (H1-H2). Let $\underline{u}, \bar{u} \in$ $W^{1, p}(\Omega) \cap L^{\infty}(\Omega)$ be, respectively, a sub-solution and a super-solution of problem (2.1), with $\underline{u}(x) \leq \bar{u}(x)$ a.e. in $\Omega$. Then there exists a minimal (and, respectively, a maximal) weak solution $u_{*}$ (resp. $u^{*}$ ) for problem (2.1) in the "interval"

$$
[\underline{u}, \bar{u}]=\left\{u \in L^{\infty}(\Omega): \underline{u}(x) \leq u(x) \leq \bar{u}(x) \text { a.e. in } \Omega\right\} .
$$

In particular, every weak solution $u \in[\underline{u}, \bar{u}]$ of (2.1) satisfies also $u_{*}(x) \leq u(x) \leq$ $u^{*}(x)$ for a.e. $x \in \Omega$. 
Proof. Consider the set $[\underline{u}, \bar{u}]$ with the topology of convergence a.e., and define the operator $S:[\underline{u}, \bar{u}] \rightarrow L^{p^{\prime}}(\Omega)$ by

$$
S v=\widetilde{h}(\cdot, v(\cdot))+g(v(\cdot)) \in L^{\infty}(\Omega) \subset L^{p^{\prime}}(\Omega), \forall v \in[\underline{u}, \bar{u}] .
$$

By using hypotheses (H1-H2), we get that $S$ is nondecreasing and bounded. Moreover, if $v_{n}, v \in[\underline{u}, \bar{u}]$, then

$$
\left\|S v_{n}-S v\right\|_{L^{p^{\prime}}}^{p^{\prime}}=\int_{\Omega}\left|\widetilde{h}\left(x, v_{n}\right)+g\left(v_{n}\right)-\widetilde{h}(x, v)-g(v)\right|^{p^{\prime}} .
$$

Let $v_{n} \rightarrow v$ a.e. in $\Omega$. Applying the Lebesgue dominated convergence theorem, we obtain that $\left\|S v_{n}-S v\right\|_{L^{p^{\prime}}} \rightarrow 0$, and then $S$ is continuous.

Consider the continuous nondecreasing operator $F:[\underline{u}, \bar{u}] \rightarrow W_{0}^{1, p}(\Omega)$ defined by $F=T \circ S$, i.e., for a function $v \in[\underline{u}, \bar{u}], F(v)$ is the unique weak solution of the boundary value problem

$$
\begin{array}{cl}
-\Delta_{p} u(x)+g(u(x))=\widetilde{h}(x, v(x))+g(v(x)), & x \in \Omega, \\
u(x)=0, & x \in \partial \Omega .
\end{array}
$$

Writing $u_{1}=F(\underline{u}), u^{1}=F(\bar{u})$, we obtain that $\forall \phi \in W_{0}^{1, p}(\Omega), \phi \geq 0$,

$$
\begin{aligned}
\int_{\Omega}\left|\nabla u_{1}\right|^{p-2} \nabla u_{1} \cdot \nabla \phi+\int_{\Omega} g\left(u_{1}\right) \phi & =\int_{\Omega} \widetilde{h}(x, \underline{u}) \phi+\int_{\Omega} g(\underline{u}) \phi \\
& \geq \int_{\Omega}|\nabla \underline{u}|^{p-2} \nabla \underline{u} \cdot \nabla \phi+\int_{\Omega} g(\underline{u}) \phi,
\end{aligned}
$$

and

$$
\begin{aligned}
\int_{\Omega}\left|\nabla u^{1}\right|^{p-2} \nabla u^{1} \cdot \nabla \phi+\int_{\Omega} g\left(u^{1}\right) \phi & =\int_{\Omega} \widetilde{h}(x, \bar{u}) \phi+\int_{\Omega} g(\bar{u}) \phi \\
& \leq \int_{\Omega}|\nabla \bar{u}|^{p-2} \nabla \bar{u} \cdot \nabla \phi+\int_{\Omega} g(\bar{u}) \phi .
\end{aligned}
$$

Applying Lemma 2.2 and taking into account that $F$ is nondecreasing, we obtain $\underline{u} \leq F(\underline{u}) \leq F(u) \leq F(\bar{u}) \leq \bar{u}$, a.e. in $\Omega, \forall u \in[\underline{u}, \bar{u}]$. Repeating the same reasoning, we can prove the existence of sequences $\left\{u^{n}\right\}$ and $\left\{u_{n}\right\}$ satisfying

$$
\begin{array}{ll}
u^{0}=\bar{u}, & u^{n+1}=F\left(u^{n}\right), \\
u_{0}=\underline{u}, & u_{n+1}=F\left(u_{n}\right),
\end{array}
$$

and, for every weak solution $u \in[\underline{u}, \bar{u}]$ of problem (2.1), we have

$$
u_{0} \leq u_{1} \leq \ldots \leq u_{n} \leq u \leq u^{n} \leq \ldots \leq u^{1} \leq u^{0} \text { a.e. in } \Omega .
$$

Then, $u_{n} \rightarrow u_{*}, u^{n} \rightarrow u^{*}$, a.e. in $\Omega$, with $u_{*}, u^{*} \in[\underline{u}, \bar{u}], u_{*} \leq u^{*}$ a.e. in $\Omega$. Since $u_{n+1}=F\left(u_{n}\right) \rightarrow F\left(u_{*}\right)$, and $u^{n+1}=F\left(u^{n}\right) \rightarrow F\left(u^{*}\right)$ in $W_{0}^{1, p}(\Omega)$ by continuity of $F$, then $u_{*}, u^{*} \in W_{0}^{1, p}(\Omega)$ with $u_{*}=F\left(u_{*}\right), u^{*}=F\left(u^{*}\right)$. This completes the proof.

Remark. Note that it is sufficient for function $\widetilde{h}$ to be defined in the set $\bar{\Omega} \times$ $\left[\right.$ ess $\inf _{x \in \bar{\Omega}} \underline{u}(x)$, ess $\left.\sup _{x \in \bar{\Omega}} \bar{u}(x)\right]$, and the function $s \mapsto g(s)+\widetilde{h}(x, s)$ must be nondecreasing only while $s \in[\underline{u}(x), \bar{u}(x)]$. 
Definition 2.5. Consider a function $\mu(x) \in L^{\infty}(\Omega)$, and the following eigenvalue problem:

$$
\begin{array}{cl}
-\Delta_{p} u(x)+\mu(x)|u(x)|^{p-2} u(x)=\lambda|u(x)|^{p-2} u(x), & x \in \Omega, \\
u(x)=0, & x \in \partial \Omega .
\end{array}
$$

We denote by $\lambda_{1}(\Omega, \mu)$ the principal eigenvalue of this eigenvalue problem. It is known (see [1], [10]) that it is simple, isolated, and can be expressed as

$$
\lambda_{1}(\Omega, \mu)=\inf _{u \in W_{0}^{1, p}(\Omega) \backslash\{0\}} \frac{\int_{\Omega}|\nabla u|^{p}+\int_{\Omega} \mu|u|^{p}}{\int_{\Omega}|u|^{p}} .
$$

The associated eigenfunction $\phi_{1}(\Omega, \mu)$ (a regular function where the infimum of the Rayleigh-type quotient is attained) can be chosen strictly positive in $\Omega$, with $\left\|\phi_{1}(\Omega, \mu)\right\|_{L^{\infty}(\Omega)}=1$.

Proposition 2.6. The eigenvalue $\lambda_{1}(\Omega, \mu)$ has the following properties:

i) $\lambda_{1}(\Omega, \mu)$ is decreasing with respect to the domain $\Omega$ (we consider the ordering given by the inclussion of domains).

ii) $\lambda_{1}(\Omega, \mu)$ is increasing with respect to the weight function $\mu$.

iii) $\forall M \in \mathbb{R}, \lambda_{1}(\Omega, \mu+M)=\lambda_{1}(\Omega, \mu)+M$.

iv) $\forall t \in(0,1)$, and $\mu_{1}, \mu_{2} \in L^{\infty}(\Omega)$,

$$
\lambda_{1}\left(\Omega, t \mu_{1}+(1-t) \mu_{2}\right) \geq t \lambda_{1}\left(\Omega, \mu_{1}\right)+(1-t) \lambda_{1}\left(\Omega, \mu_{2}\right) .
$$

v) $\lambda_{1}(\Omega, \mu)$ is continuous with respect to $\mu \in L^{\infty}(\Omega)$.

Proof. i) Consider $\Omega_{1} \subset \Omega_{2}$, two bounded and regular domains in $\mathbb{R}^{N}$, and let $\widetilde{u} \in W_{0}^{1, p}\left(\Omega_{2}\right)$ be defined as the zero extension of $\phi_{1}=\phi_{1}\left(\Omega_{1}, \mu\right)$ outside of $\Omega_{1}$. It is clear that

$$
\lambda_{1}\left(\Omega_{2}, \mu\right) \leq \frac{\int_{\Omega_{2}}\left(|\nabla \widetilde{u}|^{p}+\mu|\widetilde{u}|^{p}\right)}{\int_{\Omega_{2}}|\widetilde{u}|^{p}}=\frac{\int_{\Omega_{1}}\left(\left|\nabla \phi_{1}\right|^{p}+\mu\left|\phi_{1}\right|^{p}\right)}{\int_{\Omega_{1}}\left|\phi_{1}\right|^{p}}=\lambda_{1}\left(\Omega_{1}, \mu\right) .
$$

Moreover, if $\Omega_{1} \neq \Omega_{2}$, then $\widetilde{u}$ is not strictly positive in $\Omega_{2}$, and so $\widetilde{u}$ is not an eigenfunction associated with $\lambda_{1}\left(\Omega_{2}, \mu\right)$. Consequently, the previous inequality must be strict.

ii) is a consequence of the variational characterization of $\lambda_{1}(\Omega, \mu)$.

iii) We have

$$
\begin{aligned}
\lambda_{1}(\Omega, \mu+M) & =\min _{u \in W_{0}^{1, p}(\Omega) \backslash\{0\}} \frac{\int_{\Omega}\left(|\nabla u|^{p}+(\mu+M)|u|^{p}\right)}{\int_{\Omega}|u|^{p}} \\
& =\min _{u \in W_{0}^{1, p}(\Omega) \backslash\{0\}}\left\{\frac{\int_{\Omega}\left(|\nabla u|^{p}+\mu|u|^{p}\right)}{\int_{\Omega}|u|^{p}}+M\right\} \\
& =\lambda_{1}(\Omega, \mu)+M .
\end{aligned}
$$


iv) We have

$$
\begin{gathered}
\lambda_{1}\left(\Omega, t \mu_{1}+(1-t) \mu_{2}\right) \\
=\min _{u \in W_{0}^{1, p}(\Omega) \backslash\{0\}} \frac{\int_{\Omega}\left(|\nabla u|^{p}+\left(t \mu_{1}+(1-t) \mu_{2}\right)|u|^{p}\right)}{\int_{\Omega}|u|^{p}} \\
=\min _{u \in W_{0}^{1, p}(\Omega) \backslash\{0\}}\left\{\frac{t \int_{\Omega}\left(|\nabla u|^{p}+\mu_{1}|u|^{p}\right)}{\int_{\Omega}|u|^{p}}+\frac{(1-t) \int_{\Omega}\left(|\nabla u|^{p}+\mu_{2}|u|^{p}\right)}{\int_{\Omega}|u|^{p}}\right\} \\
\geq t \lambda_{1}\left(\Omega, \mu_{1}\right)+(1-t) \lambda_{1}\left(\Omega, \mu_{2}\right) . \\
\text { v) Setting } \varphi=\frac{\phi_{1}(\Omega, \mu)}{\left\|\phi_{1}(\Omega, \mu)\right\|_{L^{p}}} \text { and } \varphi_{n}=\frac{\phi_{1}\left(\Omega, \mu_{n}\right)}{\left\|\phi_{1}\left(\Omega, \mu_{n}\right)\right\|_{L^{p}}}, \forall n \in \mathbb{N}, \text { we obtain } \\
\lambda_{1}(\Omega, \mu)=\int_{\Omega}\left(|\nabla \varphi|^{p}+\mu|\varphi|^{p}\right) ; \quad \lambda_{1}\left(\Omega, \mu_{n}\right)=\int_{\Omega}\left(\left|\nabla \varphi_{n}\right|^{p}+\mu_{n}\left|\varphi_{n}\right|^{p}\right) .
\end{gathered}
$$

Observe that

$$
\begin{gathered}
\lambda_{1}(\Omega, \mu)+\int_{\Omega}\left[\mu_{n}-\mu\right]|\varphi|^{p}=\int_{\Omega}\left(|\nabla \varphi|^{p}+\mu_{n}|\varphi|^{p}\right) \\
\geq \lambda_{1}\left(\Omega, \mu_{n}\right) \\
=\int_{\Omega}\left(\left|\nabla \varphi_{n}\right|^{p}+\mu\left|\varphi_{n}\right|^{p}+\left[\mu_{n}-\mu\right]\left|\varphi_{n}\right|^{p}\right) \geq \lambda_{1}(\Omega, \mu)+\int_{\Omega}\left[\mu_{n}-\mu\right]\left|\varphi_{n}\right|^{p} .
\end{gathered}
$$

Taking into account that $\left\|\mu_{n}-\mu\right\|_{L^{\infty}} \rightarrow 0$ and $\|\varphi\|_{L^{p}(\Omega)}=1,\left\|\varphi_{n}\right\|_{L^{p}(\Omega)}=1$, $\forall n \in \mathbb{N}$, we can deduce that the first and the last terms in the previous inequality converge to $\lambda_{1}(\Omega, \mu)$, concluding the proof of Lemma 2.6.

\section{THE SCALAR MODEL}

We try to find positive weak solutions of the following boundary value problem:

$$
\begin{array}{cc}
-\Delta_{p} u(x)=u(x)^{q-1} h(x, u(x)), & x \in \Omega, \\
u(x)=0, & x \in \partial \Omega,
\end{array}
$$

where $\Omega$ is a bounded and regular domain in $\mathbb{R}^{N}, p, q>1$.

Theorem 3.1. Consider problem (3.1), where h satisfies (H1-H2) and, moreover, $\exists \alpha>0$ such that $h(x, \alpha) \leq 0$, for a.e. $x \in \Omega$. Then, a sufficient condition for (3.1) to admit a nonnegative $(p>q)$ or positive $\left(p \leq q<p^{*}\right)$ weak solution is:

Case $p>q$ :

$\exists x_{0} \in \Omega, r, \varepsilon, \delta>0: h(x, s) \geq \varepsilon$ for a.e. $x \in B\left(x_{0} ; r\right) \subset \Omega, \forall s \in(0, \delta]$

(Observe that, if $h$ is continuous, this condition can be expressed as $\exists x_{0} \in$ $\Omega: h\left(x_{0}, 0\right)>0$.)

If $h(x, s) \geq 0$ for a.e. $x \in \Omega$ and for all $s \in[0, \alpha]$, then this solution is positive in $\Omega$.

Moreover, if $h$ is continuous and nonincreasing with respect to $s$, this sufficient condition is also necessary.

Case $p=q$ :

$$
\lambda_{1}(\Omega,-h(\cdot, 0))<0 .
$$

Moreover, if $h$ is nonincreasing with respect to $s$, this sufficient condition is also necessary. 
Case $p<q<p^{*}$ : $h$ is nonincreasing with respect to $s$, and

$$
\exists \gamma>0: \gamma^{p-q} \leq h(x, \gamma \beta) \text {, for a.e. } x \in \Omega,
$$

where $\beta=\|\theta\|_{L^{\infty}(\Omega)}$, and $\theta$ is any positive solution (it exists, as proved in [9]) of

$$
\begin{array}{cl}
-\Delta_{p} \theta(x)=\theta(x)^{q-1}, & x \in \Omega, \\
\theta(x)=0, & x \in \partial \Omega .
\end{array}
$$

Moreover, every such positive weak solution $u$ satisfies $u \in C_{\mathrm{loc}}^{1, \nu}(\Omega)$ with some $\nu>0$.

Proof. It is clear that the right hand side of (3.1) satisfies hypotheses (H1-H2) (set $\left.\widetilde{h}(x, s)=s^{q-1} h(x, s), \widetilde{g}(s)=s^{q-1} g(s)\right)$. Trivially, $\bar{u} \equiv \alpha>0$ is a super-solution of (3.1). In order to find an appropriate sub-solution, we consider three separate cases.

Case $p>q$ : Let $B=B\left(x_{0} ; r\right)$. Consider $\underline{u} \in W_{0}^{1, p}(\Omega)$, defined as

$$
\underline{u}(x)= \begin{cases}\gamma \phi_{1}(B, 0)(x), & x \in B, \\ 0, & x \in \Omega \backslash B,\end{cases}
$$

where $\gamma \in] 0, \delta]$ is such that $\gamma^{p-q} \leq \frac{\varepsilon}{\lambda_{1}(B, 0)}$. Observe that, $\forall s \in[0, \gamma]$, for a.e. $x \in B$,

$$
\lambda_{1}(B, 0) s^{p-1} \leq \varepsilon s^{q-1} \leq s^{q-1} h(x, s) .
$$

Then, $\forall \phi \in W_{0}^{1, p}(\Omega), \phi \geq 0$, applying the Green formula, we get

$$
\begin{aligned}
\int_{\Omega}|\nabla \underline{u}|^{p-2} \nabla \underline{u} \cdot \nabla \phi & =\int_{B}|\nabla \underline{u}|^{p-2} \nabla \underline{u} \cdot \nabla \phi \\
& =\int_{\partial B}|\nabla \underline{u}|^{p-2} \frac{\partial \underline{u}}{\partial n_{e}} \phi+\lambda_{1}(B, 0) \int_{B} \underline{u}^{p-1} \phi \\
& \leq \int_{\Omega} \underline{u}^{q-1} h(x, \underline{u}) \phi .
\end{aligned}
$$

So, $\underline{u}$ is a (nonnegative and nontrivial) sub-solution of the problem (3.1). Moreover, $\underline{u}(x) \leq \delta \leq \alpha \equiv \bar{u}(x)$, a.e. in $\Omega$.

Reciprocally, suppose that $h=h(x, s)$ is nonincreasing with respect to $s$ and continuous with respect to $x$ and $s$, and that the problem (3.1) admits a nonnegative and nontrivial weak solution $u$. Let us argue by contradiction, and suppose that $h(x, 0) \leq 0, \forall x \in \Omega$. Then

$$
\begin{array}{cl}
-\Delta_{p} u(x)=u(x)^{q-1} h(x, u(x)) \leq u(x)^{q-1} h(x, 0) \leq 0, & x \in \Omega, \\
u(x)=0, & x \in \partial \Omega .
\end{array}
$$

Applying Lemma 2.2, we can conclude that $u \leq 0$ in $\bar{\Omega}$, which is a contradiction.

Case $p=q$ : Consider $\gamma>0$ such that $g(\gamma)+\lambda_{1}(\Omega,-h(\cdot, 0)) \leq 0$. Then $\forall s \in$ $[0, \gamma]$, for a.e. $x \in \Omega$,

$$
\begin{aligned}
h(x, 0)+\lambda_{1}(\Omega,-h(\cdot, 0)) & \leq h(x, s)+g(s)+\lambda_{1}(\Omega,-h(\cdot, 0)) \\
& \leq h(x, s)+g(\gamma)+\lambda_{1}(\Omega,-h(\cdot, 0)) \\
& \leq h(x, s) .
\end{aligned}
$$


Consider $\underline{u}=\gamma \phi_{1}(\Omega,-h(\cdot, 0)) \in W_{0}^{1, p}(\Omega)$. Then, $\forall \phi \in W_{0}^{1, p}(\Omega), \phi \geq 0$, we have

$$
\begin{aligned}
\int_{\Omega}|\nabla \underline{u}|^{p-2} \nabla \underline{u} \cdot \nabla \phi & =\int_{\Omega}\left[h(x, 0)+\lambda_{1}(\Omega,-h(\cdot, 0))\right] \underline{u}^{p-1} \phi \\
& \leq \int_{\Omega} \underline{u}^{p-1} h(x, \underline{u}) \phi,
\end{aligned}
$$

i.e., $\underline{u}$ is a (positive) sub-solution of (3.1). Moreover,

$$
\begin{aligned}
g(\gamma) & \leq-\lambda_{1}(\Omega,-h(\cdot, 0)) \leq-\lambda_{1}(\Omega,-h(\cdot, \alpha)-g(\alpha)) \\
& =-\lambda_{1}(\Omega,-h(\cdot, \alpha))+g(\alpha) \leq-\lambda_{1}(\Omega, 0)+g(\alpha)<g(\alpha),
\end{aligned}
$$

so $\underline{u}(x) \leq \gamma<\alpha \equiv \bar{u}(x)$, a.e. in $\Omega$.

Reciprocally, if $h$ is nonincreasing with respect to $u$, and the problem (3.1) admits a nonnegative and nontrivial weak solution $u$, then

$$
\begin{aligned}
\lambda_{1}(\Omega,-h(\cdot, 0)) & <\frac{\int_{\Omega}|\nabla u|^{p}-\int_{\Omega} h(x, 0)|u|^{p}}{\int_{\Omega}|u|^{p}} \\
& \leq \frac{\int_{\Omega}|\nabla u|^{p}-\int_{\Omega} h(x, u) u^{p}}{\int_{\Omega} u^{p}}=0 .
\end{aligned}
$$

Case $p<q<p^{*}$ : Recall that, for $p<q<p^{*}$, there exists a positive solution $\theta$ of the problem (3.2). Put $\beta=\|\theta\|_{L^{\infty}(\Omega)}$, and let $\gamma$ be given in the hypothesis. If we consider $\underline{u}=\gamma \theta \in W_{0}^{1, p}(\Omega), \phi \in W_{0}^{1, p}(\Omega), \phi \geq 0$, then

$$
\begin{aligned}
\int_{\Omega}|\nabla \underline{u}|^{p-2} \nabla \underline{u} \cdot \nabla \phi & =\gamma^{p-1} \int_{\Omega} \theta^{q-1} \phi=\gamma^{p-q} \int_{\Omega} \underline{u}^{q-1} \phi \\
& \leq \int_{\Omega} \underline{u}^{q-1} h(x, \gamma \beta) \phi \leq \int_{\Omega} \underline{u}^{q-1} h(x, \underline{u}) \phi,
\end{aligned}
$$

i.e., $\underline{u}$ is a (nonnegative and nontrivial) sub-solution of $(3.1)$, with $\underline{u}(x) \leq$ $\gamma \beta \leq \alpha \equiv \bar{u}(x)$, a.e. in $\Omega$.

Now, in the second and the third case, the existence of a positive weak solution follows from Theorem 2.4. In the first case Theorem 2.4 implies the existence of nonnegative and nontrivial weak solution. However, if $h(x, s) \geq 0$ for a.e. $x \in \Omega$ and for all $s \in[0, \alpha]$, we can apply Harnack type inequality due to Trudinger [13, Th. 1.2], and the solution is positive in $\Omega$.

Since every weak solution $u$, the existence of which was proved in all three cases, satisfies $u \in L^{\infty}(\Omega)$, we have in fact $u \in C_{\text {loc }}^{1, \nu}(\Omega)$, on the basis of the result of Tolksdorf [12].

Examples. For $q \leq p=2$, some examples can be found in [4]. For the case $p<q<p^{*}$, consider the problem

$$
\begin{array}{cl}
-\Delta_{p} u(x)=u(x)^{q-1}[\alpha-u(x)], & x \in \Omega, \\
u(x)=0, & x \in \partial \Omega .
\end{array}
$$

Then our sufficient condition has the following form:

$$
\exists \gamma>0: \alpha-\gamma \beta-\gamma^{p-q} \geq 0,
$$


which is equivalent (maximizing that expression with respect to $\gamma$ ) to

$$
\alpha-\beta\left(\frac{q-p}{\beta}\right)^{\frac{1}{q+1-p}}-\left(\frac{q-p}{\beta}\right)^{\frac{p-q}{q+1-p}} \geq 0 .
$$

If we take $q=p+1$ (note that $p^{*}-p>1$ is necessary in this case; it holds if $p(p+1)>N)$, our condition becomes

$$
\alpha-2 \sqrt{\beta} \geq 0
$$

Now we can continue in several ways:

- Fix $\Omega \subset \mathbb{R}^{N}$ (and then $\beta$ ). We can affirm that for $\alpha$ large enough, the problem (3.3) admits a positive weak solution.

- Fix $\alpha>0$ (and then the biological species), and look for existence domains (this is a very reasonable question in biology). Let us denote $R \Omega=\{R x: x \in$ $\Omega\}$ for $R>0$. It is easy to check that if $\theta_{\Omega}$ is a solution of problem (3.2), with $\beta_{\Omega}=\left\|\theta_{\Omega}\right\|_{L^{\infty}}$, then $\theta_{R \Omega}(x)=R^{\frac{p}{p-q}} \theta_{\Omega}\left(\frac{x}{R}\right), \forall x \in R \Omega$, and $\beta_{R \Omega}=R^{\frac{p}{p-q}} \beta_{\Omega}$. In particular, $\beta_{R \Omega}=R^{-p} \beta_{\Omega}$ for $q=p+1$. So, our sufficient condition for $R \Omega$,

$$
\alpha-2 \sqrt{\beta_{R \Omega}} \geq 0
$$

can be expressed as follows:

$$
\alpha-2 R^{-p / 2} \sqrt{\beta_{\Omega}} \geq 0
$$

Then, for $R$ large enough, it is possible to obtain the existence result. This is interesting, in particular, for $\Omega=B(0 ; 1)$ (unit ball in $\mathbb{R}^{N}$ ).

In the following theorem we treat the case $p<q$ with possibly $q \geq p^{*}$.

Theorem 3.2. Consider the problem (3.1), where h satisfies (H1) and, moreover, $\exists \alpha>0$ such that $h(x, \alpha)=0, h(x, s)>0$ for $0<s<\alpha$ and for a.e. $x \in \Omega$. Let $H: \Omega \times \mathbb{R} \rightarrow \mathbb{R}$ be defined by

$$
H(x, t)= \begin{cases}0, & \text { if } t<0, x \in \Omega, \\ \int_{0}^{t} s^{q-1} h(x, s) d s & \text { if } 0 \leq t \leq \alpha, x \in \Omega, \\ H(x, \alpha), & \text { if } t>\alpha, x \in \Omega .\end{cases}
$$

Then the following implication holds:

$$
\sup _{u \in W_{0}^{1, p}(\Omega) \backslash\{0\}} \frac{\int_{\Omega} H(x, u)}{\|u\|_{W_{0}^{1, p}}^{p}} \geq \frac{1}{p} \Longrightarrow \begin{aligned}
& \text { (3.1) admits a positive } \\
& \text { weak solution } u \in C_{\mathrm{loc}}^{1, \nu}(\Omega) .
\end{aligned}
$$

Reciprocally, if $h=h(x, s)$ is a nonincreasing function with respect to $s$, then

$$
\begin{aligned}
& \text { (3.1) admits a nonnegative } \\
& \text { and nontrivial weak solution }
\end{aligned} \Longrightarrow \sup _{u \in W_{0}^{1, p}(\Omega) \backslash\{0\}} \frac{\int_{\Omega} H(x, u)}{\|u\|_{W_{0}^{1, p}}^{p}} \geq \frac{1}{q} .
$$

Proof. Observe that $H=H(x, t)$ is nondecreasing with respect to $t$. Denote by $M>0$ the constant for which $|h(x, s)| \leq M$ for any $s \in[0, \alpha]$ and for a.e. $x \in \Omega$. 
Then for $t_{1}, t_{2} \in \mathbb{R}, t_{1}<t_{2}$, and for a.e. $x \in \Omega$, we have

$$
\begin{aligned}
\left|H\left(x, t_{2}\right)-H\left(x, t_{1}\right)\right| & \leq \int_{\max \left\{0, t_{1}\right\}}^{\min \left\{\alpha, t_{2}\right\}} s^{q-1}|h(x, s)| d s \\
& \leq \frac{M}{q}\left[\left(\min \left\{\alpha, t_{2}\right\}\right)^{q}-\left(\max \left\{0, t_{1}\right\}\right)^{q}\right] \\
& \leq L\left|t_{2}-t_{1}\right|,
\end{aligned}
$$

where $L>0$ depends only on $M, q$ and $\alpha$. Hence, $H(x, \cdot)$ is a Lipschitzian function and the constant $L$ is uniform for a.e. $x \in \Omega$.

Let $\Phi: W_{0}^{1, p}(\Omega) \rightarrow \mathbb{R}$ be defined as follows:

$$
\Phi(u)= \begin{cases}\frac{\int_{\Omega} H(x, u)}{\|u\|^{p}}, & \text { if } u \neq \equiv, \\ 0, & \text { if } u \equiv 0,\end{cases}
$$

where $\|u\|=\|u\|_{W_{0}^{1, p}}$. As $H$ is bounded, it is clear that $\lim _{\|u\| \rightarrow+\infty} \Phi(u)=0$. In order to calculate $\lim _{\|u\| \rightarrow 0} \Phi(u)$, take $r \in\left(p, \min \left\{p^{*}, q\right\}\right)$. Then, if $t \in[0, \alpha], x \in \Omega$,

$$
H(x, t) \leq \frac{M}{q} t^{q}=\frac{M}{q} t^{q-r} t^{r} \leq \frac{M}{q} \alpha^{q-r} t^{r} .
$$

If $t>\alpha, x \in \Omega$,

$$
H(x, t)=H(x, \alpha) \leq \frac{M}{q} \alpha^{q-r} \alpha^{r}<\frac{M}{q} \alpha^{q-r} t^{r} .
$$

So, $H(x, t) \leq \frac{M}{q} \alpha^{q-r}|t|^{r}, \forall t \in \mathbb{R}$ and for a.e. $x \in \Omega$. Taking into account that $W_{0}^{1, p}(\Omega) \subset L^{r}(\Omega)$, we obtain

$$
0 \leq \Phi(u)=\frac{\int_{\Omega} H(x, u)}{\|u\|^{p}} \leq \frac{\frac{M}{q} \alpha^{q-r} \int_{\Omega}|u|^{r}}{\|u\|^{p}} \leq C \frac{\|u\|^{r}}{\|u\|^{p}}=C\|u\|^{r-p} .
$$

Then $\lim _{\|u\| \rightarrow 0} \Phi(u)=0$, and so $\Phi$ is bounded on $W_{0}^{1, p}(\Omega)$. Let $S_{H}=\sup _{u \in W_{0}^{1, p}(\Omega)} \Phi(u)$, and consider $\left\{u_{n}\right\} \subset W_{0}^{1, p}(\Omega)$ such that $\Phi\left(u_{n}\right) \rightarrow S_{H}$ on $W_{0}^{1, p}(\Omega)$. It is clear that $\left\{u_{n}\right\}$ is bounded in $W_{0}^{1, p}(\Omega)$. Moreover, we can suppose that $\left\|u_{n}\right\| \rightarrow \rho>0$. Then, there exists a subsequence $u_{n_{k}} \rightarrow u_{0}$ weakly in $W_{0}^{1, p}(\Omega)$, with $\left\|u_{0}\right\| \leq \rho$. Moreover, as $W_{0}^{1, p}(\Omega)$ is compactly embedded into $L^{1}(\Omega)$, we have $u_{n_{k}} \rightarrow u_{0}$ in $L^{1}(\Omega)$. Moreover, since $H=H(x, \cdot)$ is Lipschitzian with constant $L>0$, we have

$$
\left|\int_{\Omega} H\left(x, u_{n_{k}}\right)-\int_{\Omega} H\left(x, u_{0}\right)\right| \leq L \int_{\Omega}\left|u_{n_{k}}-u_{0}\right|
$$

and so $\int_{\Omega} H\left(x, u_{n_{k}}\right) \rightarrow \int_{\Omega} H\left(x, u_{0}\right)$. Hence,

$$
S_{H}=\lim _{n \rightarrow+\infty} \Phi\left(u_{n_{k}}\right)=\frac{\int_{\Omega} H\left(x, u_{0}\right)}{\rho^{p}} \leq \Phi\left(u_{0}\right) .
$$


Then, $\Phi\left(u_{0}\right)=S_{H}$ and $\left\|u_{0}\right\|=\rho$. As $\Phi$ is differentiable at $W_{0}^{1, p}(\Omega) \backslash\{0\}$, we obtain that $\Phi^{\prime}\left(u_{0}\right) \equiv 0$, i.e., $\forall v \in W_{0}^{1, p}(\Omega)$,

$$
0=\Phi^{\prime}\left(u_{0}\right)(v)=\frac{\left\|u_{0}\right\|^{p} \int_{\Omega} H_{u}^{\prime}\left(x, u_{0}\right) v-\int_{\Omega} H\left(x, u_{0}\right) \int_{\Omega} p\left|\nabla u_{0}\right|^{p-2} \nabla u_{0} \cdot \nabla v}{\left\|u_{0}\right\|^{2 p}} .
$$

This means that $u_{0}$ is a weak solution of the problem

$$
\begin{array}{cl}
-\Delta_{p} u(x)=\frac{1}{p S_{H}} H_{u}^{\prime}(x, u(x)), & x \in \Omega, \\
u(x)=0, & x \in \partial \Omega .
\end{array}
$$

Moreover, observe that, due to the definition of $H$, we have

$$
\left\|u_{0}^{-}\right\|^{p}=\int_{\Omega}\left|\nabla u_{0}\right|^{p-2} \nabla u_{0} \cdot \nabla u_{0}^{-}=\frac{1}{p S_{H}} \int_{\Omega} H_{u}^{\prime}\left(x, u_{0}\right) u_{0}^{-}=0
$$

and

$$
\begin{aligned}
\left\|\left(u_{0}-\alpha\right)^{+}\right\|^{p} & =\int_{\Omega}\left|\nabla u_{0}\right|^{p-2} \nabla u_{0} \cdot \nabla\left(u_{0}-\alpha\right)^{+} \\
& =\frac{1}{p S_{H}} \int_{\Omega} H_{u}^{\prime}\left(x, u_{0}\right)\left(u_{0}-\alpha\right)^{+}=0,
\end{aligned}
$$

i.e. $0 \leq u_{0}(x) \leq \alpha$ for a.e. $x \in \Omega$. Hence, $u_{0}$ is a nonnegative weak solution of the problem

$$
\begin{array}{cc}
-\Delta_{p} u(x)=\frac{1}{p S_{H}} u(x)^{q-1} h(x, u(x)), & x \in \Omega, \\
u(x)=0, & x \in \partial \Omega .
\end{array}
$$

Our hypothesis is just that $\frac{1}{p S_{H}} \leq 1$. Then $u_{0}$ becomes a (nonnegative and nontrivial) sub-solution of problem (3.1). On the other hand we have also the supersolution $\bar{u} \equiv \alpha \geq u_{0}$ a.e. in $\Omega$. Applying Theorem 2.4, we deduce the existence of the desired weak solution. The local $C^{1, \nu}$-regularity of the weak solution follows by the same reasoning as in the proof of Theorem 3.1. The positivity of the solution follows now from the result of Trudinger [13, Th. 1.1].

Reciprocally, if $h=h(x, s)$ is a nonincreasing function with respect to $s$, observe that $\forall t \geq 0$, for a.e. $x \in \Omega$,

$$
H(x, t) \geq \int_{0}^{t} s^{q-1} h(x, s) d s \geq h(x, t) \int_{0}^{t} s^{q-1} d s=\frac{t^{q}}{q} h(x, t) .
$$

Then, if (3.1) admits a nonnegative and nontrivial weak solution $u$, this solution verifies $\int_{\Omega}|\nabla u|^{p}=\int_{\Omega} u^{q} h(x, u)$, and

$$
\Phi(u)=\frac{\int_{\Omega} H(x, u)}{\int_{\Omega}|\nabla u|^{p}} \geq \frac{\int_{\Omega} \frac{u^{q}}{q} h(x, u)}{\int_{\Omega} u^{q} h(x, u)}=\frac{1}{q},
$$

and the proof is completed. 
Example. Fix $\alpha>0,1<p<q$, and apply the result to the problem

$$
\left\{\begin{array}{cl}
-\Delta_{p} u(x)=u(x)^{q-1} \lambda[\alpha-u(x)], & x \in \Omega, \\
u(x)=0, & x \in \partial \Omega .
\end{array}\right.
$$

In order to emphasize the dependence on $\lambda>0$, we write $\Phi_{\lambda}$ instead of $\Phi$ for the functional associated with $\left(P_{\lambda}\right)$ (see the proof of Theorem 3.2). Then $\Phi_{\lambda}=\lambda \Phi_{1}$ and we can affirm that for $\lambda>0$ large enough, $\left(P_{\lambda}\right)$ admits a positive weak solution, while for $\lambda>0$ small enough, $\left(P_{\lambda}\right)$ does not admit any nonnegative and nontrivial weak solution.

We must mention that this type of example (and also Theorem 3.2), for $2=$ $p<q$, has been obtained, by using variational techniques, in [2], where at least two solutions are found. In this case, the variational nature of the two solutions is one of minimum-type and one of mountain pass-type. It is known (see, for instance, [3]) that between any sub- and super-solutions which are not solutions, there always exists a local minimum of the associated variational operator. Then we can affirm that a minimum-type solution lies between our sub-solution and our super-solution, while the mountain pass-type solution seems not to be approachable by our method. However, we find an iterative scheme which provides a monotone convergent sequence to solutions of $\left(P_{\lambda}\right)$. Moreover, as we show in the next section, the proof of Theorem 3.2 can be extended to systems of equations which, in general, do not have a variational structure.

\section{The STUdy OF SYSTEMS}

In this section we study the existence of solutions $(u, v)$ of the system

$$
\begin{array}{cc}
-\Delta_{p} u(x)=u(x)^{q-1} h(x, u(x), v(x)), & x \in \Omega, \\
-\Delta_{p} v(x)=v(x)^{q-1} k(x, u(x), v(x)), & x \in \Omega, \\
u(x)=v(x)=0, & x \in \partial \Omega,
\end{array}
$$

where $\Omega$ is a bounded and regular domain in $\mathbb{R}^{N}, p, q$ are real constants, $p, q>1$, and $h, k: \bar{\Omega} \times \mathbb{R}^{2} \rightarrow \mathbb{R}$ satisfy

(HK1)

$h(x, s, t)$ and $k(x, s, t)$ are Carathéodory functions (i.e. $h(x, \cdot, \cdot)$, $k(x, \cdot, \cdot)$ are continuous for a.e. $x \in \Omega$ and $h(\cdot, s, t), k(\cdot, s, t)$ are measurable for all $\left.(s, t) \in \mathbb{R}^{2}\right)$, and $h(\cdot, s, t), k(\cdot, s, t)$ are bounded if $s$ and $t$ belong to bounded sets.

(HK2)

$\exists g: \mathbb{R} \rightarrow \mathbb{R}$, continuous and increasing, such that $g(0)=0$, and the maps $s \mapsto h(x, s, t)+g(s)$ and $t \mapsto k(x, s, t)+g(t)$ are nondecreasing for a.e. $x \in \Omega$.

Due to the origin of the problem (population dynamics), we are interested in finding a weak solution $(u, v)$ of $(4.1)$ such that both components, $u$ and $v$, are nonnegative and nontrivial (such a solution $(u, v)$ is called a "coexistence state" for (4.1)).

In order to obtain coexistence results for systems like (4.1), we have to employ an extension of the classical method of sub- and super-solutions, applicable to general 
systems of the form

$$
\begin{array}{cc}
-\Delta_{p} u(x)=\widetilde{h}(x, u(x), v(x)), & x \in \Omega, \\
-\Delta_{p} v(x)=\widetilde{k}(x, u(x), v(x)), & x \in \Omega, \\
u(x)=v(x)=0, & x \in \partial \Omega,
\end{array}
$$

where $\Omega$ is a bounded and regular domain in $\mathbb{R}^{N}, p>1$, and the nonlinearities $\widetilde{h}, \widetilde{k}: \bar{\Omega} \times \mathbb{R}^{2} \rightarrow \mathbb{R}$ satisfy (HK1-HK2).

Definition 4.1. Let $\underline{u}, \bar{u}, \underline{v}, \bar{v} \in W^{1, p}(\Omega) \cap L^{\infty}(\Omega)$. These functions are said to be a system of sub-super-solutions for system (4.2) if they satisfy

a)

$$
\left\{\begin{array}{cc}
\underline{u}(x) \leq \bar{u}(x), & \underline{v}(x) \leq \bar{v}(x), \quad \text { a.e. in } \Omega \\
\underline{u} \leq 0 \leq \bar{u}, \quad \underline{v} \leq 0 \leq \bar{v}, \quad \text { on } \partial \Omega
\end{array}\right.
$$

b) $\forall \phi \in W_{0}^{1, p}(\Omega), \phi \geq 0$,

$$
\begin{aligned}
& \forall v \in[\underline{v}, \bar{v}],\left\{\begin{array}{l}
\int_{\Omega}|\nabla \bar{u}|^{p-2} \nabla \bar{u} \cdot \nabla \phi \geq \int_{\Omega} \widetilde{h}(x, \bar{u}, v) \phi, \\
\int_{\Omega}|\nabla \underline{u}|^{p-2} \nabla \underline{u} \cdot \nabla \phi \leq \int_{\Omega} \widetilde{h}(x, \underline{u}, v) \phi,
\end{array}\right. \\
& \forall u \in[\underline{u}, \bar{u}],\left\{\begin{array}{l}
\int_{\Omega}|\nabla \bar{v}|^{p-2} \nabla \bar{v} \cdot \nabla \phi \geq \int_{\Omega} \widetilde{k}(x, u, \bar{v}) \phi, \\
\int_{\Omega}|\nabla \underline{v}|^{p-2} \nabla \underline{v} \cdot \nabla \phi \leq \int_{\Omega} \widetilde{k}(x, u, \underline{v}) \phi .
\end{array}\right.
\end{aligned}
$$

Theorem 4.2. Consider system (4.2) under the hypotheses (HK1-HK2). Let us suppose that $\exists \underline{u}, \bar{u}, \underline{v}, \bar{v} \in W^{1, p}(\Omega) \cap L^{\infty}(\Omega)$, a system of sub-super-solutions for (4.2). Then there exists a weak solution of (4.2),

$$
(u, v) \in[\underline{u}, \bar{u}] \times[\underline{v}, \bar{v}] .
$$

The proof of Theorem 4.2 is the standard adaptation of the associated result for the case of scalar equations to systems. Similar proofs for $p=2$ can be found in [5], [7], [8].

As an application of the previous general result, we obtain sufficient conditions for system (4.1) to admit a positive coexistence state.

Theorem 4.3. Consider system (4.1), where functions $h, k: \bar{\Omega} \times \mathbb{R}^{2} \rightarrow \mathbb{R}$ satisfy hypotheses (HK1-HK2) and, moreover, $\exists \alpha_{1}, \alpha_{2}$ such that $h\left(x, \alpha_{1}, t\right) \leq 0, \forall t \in$ $\left[0, \alpha_{2}\right]$, for a.e. $x \in \Omega$, and $k\left(x, s, \alpha_{2}\right) \leq 0, \forall s \in\left[0, \alpha_{1}\right]$, for a.e. $x \in \Omega$. Then, a sufficient condition for (4.1) to admit a coexistence state $(p>q)$ or positive coexistence state $\left(p \leq q<p^{*}\right)$ is:

Case $p>q: \exists x_{1}, x_{2} \in \Omega, \exists r_{1}, r_{2}, \varepsilon_{1}, \varepsilon_{2}, \delta_{1}, \delta_{2}>0$ such that

$$
\begin{aligned}
& \quad h(x, s, t) \geq \varepsilon_{1} \text {, for a.e. } x \in B\left(x_{1} ; r_{1}\right) \subset \Omega, \forall s \in\left(0, \delta_{1}\right], \forall t \in\left[0, \alpha_{2}\right], \\
& \text { and } \\
& \quad k(x, s, t) \geq \varepsilon_{2} \text {, for a.e. } x \in B\left(x_{2} ; r_{2}\right) \subset \Omega, \forall t \in\left(0, \delta_{2}\right], \forall s \in\left[0, \alpha_{1}\right] .
\end{aligned}
$$$$
\text { If } h(x, s, t) \geq 0, k(x, s, t) \geq 0 \text { for a.e. } x \in \Omega \text { and for all }(s, t) \in\left[0, \alpha_{1}\right] \times\left[0, \alpha_{2}\right] \text {, }
$$
the coexistence state is positive. 
Case $p=q$ :

$$
\exists \varepsilon>0:\left\{\begin{array}{l}
h(x, 0, t)>\lambda_{1}(\Omega, 0)+\varepsilon, \quad \text { for a.e. } x \in \Omega, \forall t \in\left[0, \alpha_{2}\right], \\
k(x, s, 0)>\lambda_{1}(\Omega, 0)+\varepsilon, \quad \text { for a.e. } x \in \Omega, \forall s \in\left[0, \alpha_{1}\right] .
\end{array}\right.
$$

Case $p<q<p^{*}$ : The functions $s \mapsto h(x, s, t)$ and $t \mapsto k(x, s, t)$ are nonincreasing, and

$$
\exists \gamma_{1}, \gamma_{2}>0:\left\{\begin{array}{l}
\gamma_{1}^{p-q} \leq h\left(x, \gamma_{1} \beta, t\right), \text { for a.e. } x \in \Omega, \forall t \in\left(0, \alpha_{2}\right], \\
\gamma_{2}^{p-q} \leq k\left(x, s, \gamma_{2} \beta\right), \text { for a.e. } x \in \Omega, \forall s \in\left(0, \alpha_{1}\right],
\end{array}\right.
$$

where $\beta$ is defined as in Theorem 3.1.

Moreover, the coexistence state $(u, v)$ satisfies $u, v \in C_{\mathrm{loc}}^{1, \nu}(\Omega)$.

Proof. Using the same ideas as in Theorem 3.1, it is easy to prove that $\underline{u}, \bar{u}, \underline{v}, \bar{v} \in$ $W^{1, p}(\Omega) \cap L^{\infty}(\Omega)$ form a system of sub-super-solutions for system (4.1), if those functions are defined as follows: $\bar{u} \equiv \alpha_{1}>0, \bar{v} \equiv \alpha_{2}>0$, and

Case $p>q$ : Let $B_{1}=B\left(x_{1} ; r_{1}\right), B_{2}=B\left(x_{2} ; r_{2}\right)$; then

$$
\begin{aligned}
& \underline{u}(x)= \begin{cases}\gamma_{1} \phi_{1}\left(B_{1}, 0\right)(x), & x \in B_{1}, \\
0, & x \in \Omega \backslash B_{1},\end{cases} \\
& \underline{v}(x)= \begin{cases}\gamma_{2} \phi_{1}\left(B_{2}, 0\right)(x), & x \in B_{2}, \\
0, & x \in \Omega \backslash B_{2},\end{cases}
\end{aligned}
$$

where $\left.\left.\gamma_{i} \in\right] 0, \delta_{i}\right]$ is such that $\gamma_{i}^{p-q} \leq \frac{\varepsilon_{i}}{\lambda_{1}\left(B_{i}, 0\right)}$, for $i=1,2$.

Case $p=q$ : Consider $\gamma>0$ such that $g(\gamma) \leq \varepsilon$; then $\underline{u}=\underline{v}=\gamma \phi_{1}(\Omega, 0)$.

Case $p<q<p^{*}$ : Consider $\theta$, a positive solution of problem (3.2), with $\beta=$ $\|\theta\|_{L^{\infty}} ;$ then $\underline{u}=\gamma_{1} \theta, \underline{v}=\gamma_{2} \theta$.

The reasoning concerning the positivity and local $C^{1, \nu}$-regularity is the same as in the proof of Theorem 3.1.

Examples. For $q \leq p=2$ see the examples in [5]. As an example for $p<q<p^{*}$, consider the particular predator-prey system with $q=p+1$ :

$$
\begin{array}{cl}
-\Delta_{p} u(x)=u(x)^{p}[a-u(x)-c v(x)], & x \in \Omega, \\
-\Delta_{p} v(x)=v(x)^{p}[e-v(x)+g u(x)], & x \in \Omega, \\
u(x)=v(x)=0, & x \in \partial \Omega,
\end{array}
$$

where $\Omega$ is a bounded and regular domain in $\mathbb{R}^{N}, p(p+1)>N$, and $a, c, e, g$ are real positive constants with $c g<1$. Consider $\alpha_{1}=a, \alpha_{2}=e+g a$. The condition of Theorem 4.3 has the following form:

$$
\exists \gamma_{1}, \gamma_{2}>0:\left\{\begin{array}{l}
\gamma_{1}^{-1} \leq a-\gamma_{1} \beta-c e-c g a, \\
\gamma_{2}^{-1} \leq e-\gamma_{2} \beta,
\end{array}\right.
$$

which is equivalent to

$$
\left\{\begin{array}{l}
e \geq 2 \sqrt{\beta} \\
a-c e-c g a \geq 2 \sqrt{\beta} .
\end{array}\right.
$$

We can now proceed similarly as in the scalar case: 
- Fix $\Omega$ (and then $\beta$ ), and take positive constants $a, c, e, g$ satisfying those conditions, or

- Fix the positive constants $a, c, e, g$, with $a-c e-c g a>0$, and consider domains as euclidean balls of sufficiently large radius, in order to obtain small values of $\beta$.

In order to treat also the case $q \geq p^{*}$ we consider the system (4.1) under slightly different assumptions on $h$ and $k$.

Theorem 4.4 (Sufficient conditions). Let $p<q$ in (4.1) and $h, k$ satisfy (HK1). Moreover, let there exist real numbers $\alpha_{H}, \widetilde{\alpha}_{H}, \alpha_{K}, \widetilde{\alpha}_{K}$ such that $0<\widetilde{\alpha}_{H} \leq \alpha_{H}$, $0<\widetilde{\alpha}_{K} \leq \alpha_{K}$ and the following conditions hold: $h\left(x, s, \alpha_{K}\right)>0$ for any $s \in$ $\left(0, \widetilde{\alpha}_{H}\right), h\left(x, \widetilde{\alpha}_{H}, \alpha_{K}\right)=0, h\left(x, s, \alpha_{K}\right) \leq h(x, s, t)$ for any $(s, t) \in\left(0, \alpha_{H}\right) \times\left(0, \alpha_{K}\right)$, $h\left(x, \alpha_{H}, t\right) \leq 0$ for any $t \in\left(0, \alpha_{K}\right) ; k(x, 0, t)>0$ for any $t \in\left(0, \widetilde{\alpha}_{K}\right), k\left(x, 0, \widetilde{\alpha}_{K}\right)=$ $0, k(x, 0, t) \leq k(x, s, t)$ for any $(s, t) \in\left(0, \alpha_{H}\right) \times\left(0, \alpha_{K}\right), k\left(x, s, \alpha_{K}\right) \leq 0$ for any $s \in\left(0, \alpha_{H}\right)$ and for a.e. $x \in \Omega$. Let $H, K: \Omega \times \mathbb{R} \rightarrow \mathbb{R}$ be defined as follows:

$$
\begin{gathered}
H(x, \sigma)= \begin{cases}0, & \text { if } \sigma<0, x \in \Omega, \\
\int_{0}^{\sigma} s^{q-1} h\left(x, s, \alpha_{K}\right) d s, & \text { if } 0 \leq \sigma \leq \widetilde{\alpha}_{H}, x \in \Omega, \\
H\left(x, \widetilde{\alpha}_{H}\right), & \text { if } \sigma>\widetilde{\alpha}_{H}, x \in \Omega ;\end{cases} \\
K(x, \tau)= \begin{cases}0, & \text { if } \tau<0, x \in \Omega, \\
\int_{0}^{\tau} t^{q-1} k(x, 0, t) d t, & \text { if } 0 \leq \tau \leq \widetilde{\alpha}_{K}, x \in \Omega, \\
K\left(x, \widetilde{\alpha}_{K}\right), & \text { if } \tau>\widetilde{\alpha}_{K}, x \in \Omega .\end{cases}
\end{gathered}
$$

Then the inequalities

$$
S_{H}=\sup _{u \in W_{0}^{1, p}(\Omega) \backslash\{0\}} \frac{\int_{\Omega} H(x, u)}{\|u\|_{W_{0}^{1, p}}^{p}} \geq \frac{1}{p}
$$

and

$$
S_{K}=\sup _{v \in W_{0}^{1, p}(\Omega) \backslash\{0\}} \frac{\int_{\Omega} K(x, v)}{\|v\|_{W_{0}^{1, p}}^{p}} \geq \frac{1}{p}
$$

are sufficient for (4.1) to admit a positive coexistence state $(u, v) \in C_{\mathrm{loc}}^{1, \nu}(\Omega)^{2}$ such that $0 \leq u \leq \alpha_{H}, 0 \leq v \leq \alpha_{K}$ in $\Omega$.

Proof. Following the lines of the proof of Theorem 3.2, we get functions $\underline{u}, \underline{v} \in$ $W_{0}^{1, p}(\Omega) \backslash\{0\}$ satisfying in the weak sense

$$
\begin{array}{cl}
-\Delta_{p} \underline{u}(x)=\frac{1}{p S_{H}} \underline{u}(x)^{q-1} h\left(x, \underline{u}(x), \alpha_{K}\right), & x \in \Omega, \\
-\Delta_{p} \underline{v}(x)=\frac{1}{p S_{K}} \underline{v}(x)^{q-1} k(x, 0, \underline{v}(x)), & x \in \Omega, \\
\underline{u}(x)=\underline{v}(x)=0, & x \in \partial \Omega,
\end{array}
$$

and, moreover, $0 \leq \underline{u}(x) \leq \widetilde{\alpha}_{H}, 0 \leq \underline{v}(x) \leq \widetilde{\alpha}_{K}$ a.e. in $\Omega$. Set $\bar{u} \equiv \alpha_{H}, \bar{v} \equiv \alpha_{K}$. Then, it follows from our hypotheses that $\underline{u}, \bar{u}, \underline{v}, \bar{v}$ is a system of sub-super-solutions for (4.1). Applying Theorem 4.2 we get the existence of a coexistence state of (4.1), $(u, v) \in[\underline{u}, \bar{u}] \times[\underline{v}, \bar{v}]$. The positivity and local $C^{1, \nu}$-regularity of $u$ and $v$ follow from [12], [13] in the same way as in the proof of Theorem 3.2. 
Theorem 4.5 (Necessary conditions). Assume the same as in Theorem 4.5 and, moreover, let $h(x, s, 0) \geq h(x, s, t)$ for any $s \geq 0, t \geq 0$ and a.e. $x \in \Omega$, let $h(x, s, 0)$ be a nonincreasing function with respect to $s$ for a.e. $x \in \Omega$, and similarly let $k(x, c, t) \geq k(x, s, t)$ for any $c>0, s \in(0, c), t \geq 0$ and a.e. $x \in \Omega$. Let $k(x, c, t)$ be a nonincreasing function with respect to $t$ for any $c>0$ and for a.e. $x \in \Omega$. Assume that (4.1) admits a coexistence state $(u, v) \in\left(W_{0}^{1, p}(\Omega) \cap L^{\infty}(\Omega)\right)^{2}$. Denote $c_{u}=\|u\|_{L^{\infty}}$ and define $\widehat{H}, \widehat{K}: \Omega \times \mathbb{R} \rightarrow \mathbb{R}$ by

$$
\begin{aligned}
& \widehat{H}(x, \sigma)= \begin{cases}0, & \text { if } \sigma<0, x \in \Omega, \\
\int_{0}^{\sigma} s^{q-1} h(x, s, 0) d s, & \text { if } 0 \leq \sigma \leq \alpha_{H}, x \in \Omega, \\
\widehat{H}\left(x, \alpha_{H}\right), & \text { if } \sigma>\alpha_{H}, x \in \Omega ;\end{cases} \\
& \widehat{K}(x, \tau)= \begin{cases}0, & \text { if } \tau<0, x \in \Omega, \\
\int_{0}^{\tau} t^{q-1} k\left(x, c_{u}, t\right) d t, & \text { if } 0 \leq \tau \leq \alpha_{K}, x \in \Omega, \\
\widehat{K}\left(x, \alpha_{K}\right), & \text { if } \tau>\alpha_{K}, x \in \Omega .\end{cases}
\end{aligned}
$$

Then

$$
S_{\widehat{H}}=\sup _{u \in W_{0}^{1, p}(\Omega) \backslash\{0\}} \frac{\int_{\Omega} \widehat{H}(x, u)}{\|u\|_{W_{0}^{1, p}}^{p}} \geq \frac{1}{q}
$$

and

$$
S_{\widehat{K}}=\sup _{v \in W_{0}^{1, p}(\Omega) \backslash\{0\}} \frac{\int_{\Omega} \widehat{K}(x, v)}{\|v\|_{W_{0}^{1, p}}^{p}} \geq \frac{1}{q} .
$$

Proof. For any $\sigma \geq 0$ and a.e. $x \in \Omega$ we have the following estimate:

$$
\widehat{H}(x, \sigma) \geq \int_{0}^{\sigma} s^{q-1} h(x, s, 0) d s \geq h(x, \sigma, 0) \int_{0}^{\sigma} s^{q-1} d s=\frac{\sigma^{q}}{q} h(x, \sigma, 0) .
$$

Then, if (4.1) admits a coexistence state $(u, v)$,

Hence

$$
\int_{\Omega}|\nabla u|^{p}=\int_{\Omega} u^{q} h(x, u, v) \leq \int_{\Omega} u^{q} h(x, u, 0) .
$$

$$
S_{\widehat{H}} \geq \frac{\int_{\Omega} \widehat{H}(x, u)}{\int_{\Omega}|\nabla u|^{p}} \geq \frac{\int_{\Omega} \frac{u^{q}}{q} h(x, u, 0)}{\int_{\Omega} u^{q} h(x, u, 0)}=\frac{1}{q} .
$$

Similarly, using the properties of $k$ and $\widehat{K}$, we derive the second inequality.

Example. Let us consider the system

$$
\begin{array}{cl}
-\Delta_{p} u(x)=\lambda u(x)^{q-1}[a-u(x)-c v(x)], & x \in \Omega, \\
-\Delta_{p} v(x)=\lambda v(x)^{q-1}[e-v(x)+g u(x)], & x \in \Omega \\
u(x)=v(x)=0, & x \in \partial \Omega
\end{array}
$$


where $\Omega$ is a bounded and regular domain in $\mathbb{R}^{N}, q>p, \lambda$ is a real positive parameter, and $a, c, e, g$ are real positive constants satisfying $a-c e-c g a>0$. In order to emphasize the dependence on the parameter $\lambda$, we denote

$$
h_{\lambda}(x, s, t)=\lambda(a-s-c t), \quad k_{\lambda}(x, s, t)=\lambda(e-t+g s) .
$$

Evidently, we have $h_{\lambda}(x, s, t)=\lambda h_{1}(x, s, t), k_{\lambda}(x, s, t)=\lambda k_{1}(x, s, t)$. The functions $h_{\lambda}, k_{\lambda}$ satisfy the assumptions of Theorem 4.4 with $\alpha_{H}=a, \alpha_{K}=e+g a, \widetilde{\alpha}_{H}=$ $a-c e-c g a, \widetilde{\alpha}_{K}=e$ (the reader is invited to sketch a figure of the nodal domains of $h_{1}$ and $k_{1}$ ). Then, it follows from Theorem 4.4 that the system (4.4) admits a positive coexistence state $(u, v) \in C_{\text {loc }}^{1, \nu}(\Omega)^{2}$ satisfying $0 \leq u \leq a, 0 \leq v \leq e+g a$ for $\lambda$ large enough (realize that $S_{H_{\lambda}}=\lambda S_{H_{1}}$ and $S_{K_{\lambda}}=\lambda S_{K_{1}}$ with $S_{H_{1}}>0, S_{K_{1}}>0$ ).

Also the assumptions of Theorem 4.5 should be verified (note that $h_{1}$ and $k_{1}$ are linear polynomials in $s$ and $t$ ). Hence the system (4.4) has not a coexistence state for $\lambda$ small enough (realize that $S_{\widehat{H}_{\lambda}}=\lambda S_{\widehat{H}_{1}}$ with $S_{\widehat{H}_{1}}>0$ ).

Remark. Note that the reasoning in the proofs of Theorems 3.2, 4.4 and 4.5 combines a variational-type technique and the method of sub- and super-solutions. This approach, in particular, enables to get existence result for systems with nonvariational structure.

Observe also that Theorems 4.4 and 4.5 should be formulated directly in terms of the example in order to avoid somewhat complicated hypotheses on $h$ and $k$. On the other hand this general formulation emphasizes the relationship between the system (4.1) and the scalar equation (3.1).

For clarity of the exposition, we restrict ourselves to the same $p$ 's and $q$ 's in the different equations in system (4.1), but it is obvious that we can deal with more general systems like

$$
\begin{array}{cc}
-\Delta_{p_{1}} u(x)=u(x)^{q_{1}-1} h(x, u(x), v(x)), & x \in \Omega, \\
-\Delta_{p_{2}} v(x)=v(x)^{q_{2}-1} k(x, u(x), v(x)), & x \in \Omega, \\
u(x)=v(x)=0, & x \in \partial \Omega,
\end{array}
$$

where $\Omega$ is a bounded and regular domain in $\mathbb{R}^{N}, p_{1}, p_{2}, q_{1}, q_{2}>1$ and $h, k$ : $\bar{\Omega} \times \mathbb{R}^{2} \rightarrow \mathbb{R}$ satisfy (HK1) and (HK2).

To do this, we must begin by giving an extension of the previous results to systems of the form

$$
\begin{array}{cc}
-\Delta_{p_{1}} u(x)=\widetilde{h}(x, u(x), v(x)), & x \in \Omega, \\
-\Delta_{p_{2}} v(x)=\widetilde{k}(x, u(x), v(x)), & x \in \Omega, \\
u(x)=v(x)=0, & x \in \partial \Omega,
\end{array}
$$

where $p_{1}, p_{2}>1$, and the nonlinearities $\widetilde{h}, \widetilde{k}: \bar{\Omega} \times \mathbb{R}^{2} \rightarrow \mathbb{R}$ satisfy (HK1-HK2).

The apropriate definition of a system of sub-super-solutions for system (4.6) is now the following.

Definition 4.6. Let $\underline{u}, \bar{u} \in W^{1, p_{1}}(\Omega) \cap L^{\infty}(\Omega), \underline{v}, \bar{v} \in W^{1, p_{2}}(\Omega) \cap L^{\infty}(\Omega)$. These functions are said to be a system of sub-super-solutions for system (4.6) if they satisfy

a)

$$
\left\{\begin{array}{cc}
\underline{u}(x) \leq \bar{u}(x), & \underline{v}(x) \leq \bar{v}(x), \quad \text { a.e. in } \Omega, \\
\underline{u} \leq 0 \leq \bar{u}, \quad \underline{v} \leq 0 \leq \bar{v}, \quad \text { on } \partial \Omega
\end{array}\right.
$$


b) $\forall \phi \in W_{0}^{1, p_{1}}(\Omega), \phi \geq 0, \forall \varphi \in W_{0}^{1, p_{2}}(\Omega), \varphi \geq 0$,

$$
\begin{aligned}
& \forall v \in[\underline{v}, \bar{v}],\left\{\begin{array}{l}
\int_{\Omega}|\nabla \bar{u}|^{p_{1}-2} \nabla \bar{u} \cdot \nabla \phi \geq \int_{\Omega} \widetilde{h}(x, \bar{u}, v) \phi, \\
\int_{\Omega}|\nabla \underline{u}|^{p_{1}-2} \nabla \underline{u} \cdot \nabla \phi \leq \int_{\Omega} \widetilde{h}(x, \underline{u}, v) \phi,
\end{array}\right. \\
& \forall u \in[\underline{u}, \bar{u}],\left\{\begin{array}{l}
\int_{\Omega}|\nabla \bar{v}|^{p_{2}-2} \nabla \bar{v} \cdot \nabla \varphi \geq \int_{\Omega} \widetilde{k}(x, u, \bar{v}) \varphi, \\
\int_{\Omega}|\nabla \underline{v}|^{p_{2}-2} \nabla \underline{v} \cdot \nabla \varphi \leq \int_{\Omega} \widetilde{k}(x, u, \underline{v}) \varphi .
\end{array}\right.
\end{aligned}
$$

Then, it is possible to prove a theorem analogous to Theorem 4.2.

Theorem 4.7. Consider system (4.6) under the hypotheses (HK1-HK2). Suppose that $\exists \underline{u}, \bar{u} \in W^{1, p_{1}}(\Omega) \cap L^{\infty}(\Omega), \underline{v}, \bar{v} \in W^{1, p_{2}}(\Omega) \cap L^{\infty}(\Omega)$, a system of sub-supersolutions for (4.6). Then there exists a weak solution of $(4.6),(u, v) \in[\underline{u}, \bar{u}] \times[\underline{v}, \bar{v}]$.

We may apply this general result to systems of type (4.5), where, for instance, $p_{1}>q_{1}$ and $p_{2}<q_{2}$ is allowed.

\section{ACKNOWLEDGMENT}

We thank the referee for some comments about the content of this paper.

\section{REFERENCES}

[1] A. Anane, Simplicité et isolation de la première valeur propre du p-laplacien avec poids, C.R. Acad. Sci. Paris, Sér. 1305 (1987), 725-728 MR 89e:35124

[2] A. Ambrosetti and P. Rabinowitz, Dual variational methods in critical point theory and applications, J. Func. Anal. 14, (1973), 349-381 MR 51:6412

[3] H. Brezis and L. Nirenberg, $H^{1}$ versus $C^{1}$ local minimizers, C.R. Acad. Sci. Paris, Sér 1317 (1993), 465-472 MR 94g:49044

[4] A. Cañada and J.L. Gámez, Some new applications of the method of lower and upper solutions to elliptic problems, Appl. Math. Lett. 6 (1993), 41-45 CMP 95:17

[5] A. Cañada and J.L. Gámez, Elliptic systems with nonlinear diffusion in population dynamics, Diff. Eqns. and Dynamical Systems. 3 (1995), 189-204 MR 97b:35064

[6] J.I. Díaz, Nonlinear partial differential equations and free boundaries, (Vol. I) Elliptic equations, London, Pitman, 1985 MR 88d:35058

[7] J. Hernández, Qualitative methods for nonlinear diffusion equations, in Nonlinear Diffusion Problems, A. Fasano and M. Primicerio (eds.), Lect. Notes in Mathematics 1224 (1986), 47-118 MR 88b:35076

[8] A. Leung and G. Fan, Existence of positive solutions for elliptic systems - degenerate and nondegenerate ecological models, J. Math. An. and Appl., 151 (1990), 512-531 MR 92e:35023

[9] M. Ôtani, Existence and nonexistence of nontrivial solutions of some nonlinear degenerate elliptic equations, J. Funct. Anal. 76 (1988), 140-159 MR 89c:35067

[10] M. Ôtani and T. Teshima, On the first eigenvalue of some quasilinear elliptic equations, Proc. Japan Acad. Ser. A 64 (1988), 8-10 MR 89h:35257

[11] M.A. Pozio and A. Tesei, Support properties of solutions for a class of degenerate parabolic problems, Comm. in Part. Diff. Eq., 12 (1987), 47-75 MR 88d:35110 
[12] P. Tolksdorf, Regularity for a more general class of quasilinear elliptic equations, J. Diff. Eq., 51 (1984), 126-150 MR 85g:35047

[13] N. Trudinger, On Harnack type inequalities and their applications to quasilinear elliptic equations, Comm. Pure Appl. Math., 20 (1967), 721-747 MR 37:1788

Department of Mathematical Analysis, University of Granada, 18071, Granada, Spain

E-mail address: acanada@goliat.ugr.es

Department of Mathematics, University of West Bohemia Plzen, Americká 42, 306 14 Plzen, Czech Republic

E-mail address: pdrabek@minea.zcu.cz

Department of Mathematical Analysis, University of Granada, 18071, Granada, Spain

E-mail address: jlgamez@goliat.ugr.es 